\title{
Recomendaciones de la Conferencia de Consenso de Bronquiolitis Aguda en España: de la evidencia a la práctica
} J. González de Dios ${ }^{a}$, C. Ochoa Sangradorb; en representación del Grupo de Trabajo
(Grupo Investigador, Grupo de Revisión y Panel de Expertos) del Proyecto aBREVIADo
(BRonquiolitis-Estudio de Variabilidad, Idoneidad y ADecuación)
aServicio de Pediatría. Hospital General Universitario de Alicante. Universidad Miguel Hernández. Alicante. España.
bServicio de Pediatría. Hospital Virgen de la Concha. Zamora. España.

\section{Resumen}

Se describe la metodología de trabajo de la Conferencia de Consenso (revisión bibliográfica, síntesis de la evidencia, método de consenso y fuerza de las recomendaciones por propuesta GRADE) y se resumen la evidencia sobre la frecuencia de bronquiolitis en la población general y en grupos de riesgo, los factores y marcadores de riesgo de formas graves, las escalas de gravedad y el perfil clínico-etiológico. Las pruebas diagnósticas habitualmente empleadas apenas resultan útiles en el manejo de la enfermedad; solo se considera necesaria la medición de la saturación de oxígeno en la valoración inicial o en el control de cambios de pacientes con compromiso respiratorio. Otras pruebas (radiografía torácica, pruebas de diagnóstico rápido de infección por virus respiratorios y pruebas de cribado de infección bacteriana) solo deben emplearse de forma muy selectiva. Existe evidencia suficiente sobre la falta de eficacia de la mayoría de las intervenciones en bronquiolitis. Al margen de la oxigenoterapia, la fluidoterapia, la aspiración de secreciones y la asistencia respiratoria, pocas opciones terapéuticas resultarán beneficiosas. Solo en los casos moderado-graves estaría justificado el ensayo con broncodilatadores inhalados (salbutamol o adrenalina) con suero salino hipertónico o sin él. En casos con fracaso respiratorio podrían emplearse heliox y técnicas de ventilación no invasiva; en pacientes con apneas valorar metilxantinas, y en pacientes graves intubados el uso de surfactante. Ningún tratamiento se ha mostrado eficaz para evitar la persistencia-recurrencia de síntomas postbronquiolitis. En cuanto a la prevención de la bronquiolitis, solo palivizumab reduce el riesgo

\footnotetext{
Javier González de Dios: javier.gonzalezdedios@gmail.com

Los autores implicados en la elaboración de este documento han realizado una declaración explícita de los conflictos de intereses por escrito. No constan conflictos de intereses que puedan influir en el contenido de este documento.

Financiado con una beca de la Fundación-Hospital Torrevieja (código de protocolo: BECA0001).

Estudio avalado por la Asociación Española de Pediatría (AEP), Asociación Española de Pediatría de Atención Primaria (AEPap), Sociedad Española de Pediatría Extrahospitalaria y Atención Primaria (SEPEAP), Sociedad Española de Urgencias Pediátricas (SEUP), Sociedad Española de Infectología Pediátrica (SEIP), Sociedad Española de Neumología Pediátrica (SENP), Sociedad Española de Inmunología Clínica y Alergia Pediátrica (SEICAP), Sociedad Española de Cuidados Intensivos Pediátricos (SECIP), Sociedad Española de Neonatología (SEN) y Sociedad Española de Cardiología Pediátrica (SECPCC).

Los datos principales del estudio han sido publicados como Conferencia de Consenso en seis artículos en Anales de Pediatría y la publicación en toda su extensión se ha publicado también como Guía de Práctica Clínica en GuiaSalud.
} 
de ingreso por virus respiratorio sincitial, aunque por su elevado coste su uso solo se justifica en pacientes de alto riesgo.

Palabras clave: Bronquiolitis viral. Revisión sistemática. Conferencia de consenso. Medicina basada en pruebas. Pediatría.

\title{
Recommendations of the Consensus Conference of Acute Bronchiolitis in Spain:
} from evidence to practice

\begin{abstract}
The methodology (literature review, evidence synthesis, method of consensus and strength of recommendations) and recommendations of the Consensus Conference are presented. The evidence on the frequency of bronchiolitis in the general population and risk groups, risk factors and markers of severe forms, scores of severity and clinical-etiological profile is summarized. The commonly used diagnostic tests are ineffective in the management of the disease; oxygen saturation measurement is considered necessary only in the initial assessment or control of changes in patients with respiratory distress. Other tests (chest radiograph, rapid diagnostic tests for respiratory virus infection and screening tests for bacterial infection) should be used only very selectively. There is sufficient evidence on the lack of effectiveness of most interventions tested in bronchiolitis. Apart from oxygen therapy, fluid therapy, aspiration of secretions and ventilation support, few treatment options will be beneficial. Only in moderate-severe bronchiolitis would be justified to test a treatment with inhaled bronchodilators (salbutamol or epinephrine) with or without hypertonic saline solution. In cases with respiratory failure could be used heliox and noninvasive ventilation techniques, methylxanthine in patients with apnea and surfactant in intubated critically ill patients. No treatment has proved effective in preventing persistence or recurrence of postbronchiolitis symptoms. Regarding the prevention of bronchiolitis, only palivizumab reduces the risk of admissions for lower respiratory infections by respiratory syncytial virus, although its high cost justifies its use only in a small group of high-risk patients.
\end{abstract}

Key words: Viral bronchiolitis. Systematic review. Consensus development conference. Evidence-based medicine. Pediatrics.

\section{Introducción}

La bronquiolitis aguda (BA) es una enfermedad que origina una gran demanda asistencial en Atención Primaria y en los Servicios de Urgencias, pues constituye la principal causa de ingreso por infección respiratoria aguda de vías bajas en el niño menor de dos años. El virus respiratorio sincitial es el agente causal dominante, aunque también están implicados otros virus.

La definición más aceptada de BA es la de McConnochie, que considera bronquiolitis el primer episodio agudo de dificultad respiratoria con sibilancias, precedido por un cuadro catarral de vías altas (rinitis, tos, con/sin fiebre), que afecta a niños menores de dos 
años, aunque preferentemente se da en el primer año de vida. Sin embargo, no todos los autores están de acuerdo con esta definición y aún no se ha llegado a un consenso acerca de los criterios necesarios para emitir el diagnóstico, especialmente los de edad o número de episodios.

Desde el punto de vista práctico, el principal problema que plantea la BA es la falta de consenso y homogeneidad en su manejo terapéutico y preventivo. Aunque existen algunos protocolos 0 guías de práctica clínica, estos presentan diferencias en la evaluación de la evidencia disponible y en sus recomendaciones. La literatura publicada en relación con el tratamiento de la BA es muy abundante, con múltiples ensayos clínicos aleatorizados (ECA) en las distintas intervenciones analizadas. La síntesis de la información de estos ECA es también sólida, fundamentada en guías de práctica clínica (GPC) y revisiones sistemáticas.

Destacamos tres GPC sobre BA, actuales y con rigurosa metodología: la de la American Academy of Pediatrics', la de la Scottish Intercollegiate Guideline Network ${ }^{2}$ y la del Cincinnati Children's Hospital Medical Center ${ }^{3}$. El repertorio de GPC National Guideline Clearinghouse acaba de publicar la sín- tesis comparativa de estas tres GPC que abordan un mismo problema de salud ${ }^{4}$. Se reconocen al menos las siguientes revisiones de la Cochrane sobre intervenciones en BA: adrenalina ${ }^{5}$, antibióti$\cos ^{6}$, beta-2-adrenérgicos ${ }^{7,8}$, bromuro de ipratropio $0^{9,10}$, corticoides ${ }^{11-14}$, fisioterapia $^{15,16}$, inmunoglobulinas ${ }^{17-19}$, ribaviri$\mathrm{na}^{20,21}$, suero salino hipertónico ${ }^{22}$ y surfactante ${ }^{23}$.

Considerando lo anteriormente expuesto, parece importante estudiar cómo se tratan las BA en nuestro medio, evaluando su variabilidad e idoneidad. Para ello llevamos a cabo el proyecto titulado "Variabilidad e idoneidad del manejo diagnóstico y terapéutico de la bronquiolitis aguda", tras elaborar específicamente unos patrones de referencia de uso apropiado de los mismos sobre la base de la revisión sistemática de la literatura y una posterior Conferencia de Consenso multidisciplinaria. En este artículo se presenta el resumen de las respuestas planteadas en la Conferencia de Consenso (con su nivel de evidencia y grado de recomendación), con especial énfasis en la clasificación de idoneidad de los procedimientos terapéuticos y preventivos en la BA. Los datos principales del estudio han sido publicados como Conferencia de Con- 
senso en seis artículos en Anales de Pediatría ${ }^{24-29}$ y la publicación en toda su extensión se ha publicado también como Guía de Práctica Clínica en GuiaSalud ${ }^{30}$.

\section{Material y métodos}

Para describir la variabilidad en el manejo de la BA se han recogido una muestra de casos pediátricos menores de dos años diagnosticados de bronquiolitis, atendidos en las consultas, Servicios de Urgencias y hospitalización de una muestra de centros de salud y hospitales españoles durante el periodo epidémico de octubre de 2007 a marzo de 2008, y con representatividad de 90 centros sanitarios de 25 provincias de España, distribuidas en 12 comunidades autónomas. Las principales características de esta muestra se han publicado recientemente ${ }^{31}$.

Para evaluar la idoneidad debíamos definir el "patrón oro" de manejo terapéutico y preventivo de la BA. Para establecer este patrón oro se han llevado a cabo los siguientes pasos:

1. Creación de un grupo de trabajo para la realización de una revisión bibliográfica sistemática y crítica sobre BA procedente de fuentes bibliográficas primarias, secundarias y terciarias, de carácter internacional (MEDLINE,
EMBASE, Cochrane Library) y nacional (Índice Médico Español). No se emplearon restricciones de idioma. No se realizaron restricciones de tiempo para los ensayos clínicos, revisiones sistemáticas y guías de práctica clínica. La búsqueda primaria de estudios observacionales se limitó a la última década en las bases de datos de MEDLINE y EMBASE, pero no en la Cochrane Library ni en el Índice Médico Español. El total de citas bibliográficas identificadas fue de 2794, de las que 953 se recuperaron y revisaron para el análisis de la evidencia.

2. Síntesis de la evidencia: todos los trabajos revisados se valoraron críticamente y se resumieron en tablas de evidencia, siguiendo una metodología explícita. En cada tabla de evidencia se detallaron los siguientes apartados: objetivo del estudio, autor y año de publicación, país de estudio, diseño y calidad del estudio, muestra de estudio e intervención, medidas de efecto y variables evaluadas, resultados (medidas de frecuencia, de efecto, de validez y de impacto), limitaciones, importancia clínica y conflictos de interés. Las tablas de evidencia se agruparon por áreas de interés (epidemiología, diagnóstico, tratamiento, prevención, pronóstico, etc.). Para cada área se elaboró un documen- 
to de revisión, con una breve introducción y justificación, un apartado con las Bases para la toma de decisiones y un conjunto de preguntas clínicas relacionadas que debían contestarse en la Conferencia de Consenso.

3. Realización de una Conferencia de Consenso, siguiendo las directrices de los National Institutes of Health ameri$\operatorname{canos}^{32}$ : revisión en profundidad de la literatura con metodología explícita por un grupo de trabajo, formación de un panel de expertos no gubernamental, planteamiento al panel de una serie de preguntas clínicas y elaboración de un documento de consenso con recomendaciones para la buena práctica clínica, y jerarquización del grado de evidencia que sustenta cada recomendación.

La constitución del panel de expertos ha seguido los siguientes criterios: 1) ser profesionales con amplia experiencia en clínica e investigación sobre bronquiolitis y prestigio en el ámbito de su especialidad; 2) proporcionar un carácter multidisciplinario al panel, y 3) procurar una representación regional equilibrada. Se ha incorporado al panel los siguientes expertos de cada una de las siguientes áreas: Atención Primaria (tres), Cardiología Pediátrica (uno), Cuidados Intensivos Pediátricos (uno), Infectología Pediátrica (uno),
Inmunología Clínica y Alergia Pediátrica (uno), Neonatología (uno), Neumología Pediátrica (uno) y Urgencias Pediátricas (dos).

Para ponderar la relevancia y aplicabilidad se considerarán medidas de efecto, impacto y validez con traducción clínica. Se propone un sistema de jerarquización de los niveles de evidencia basado en cuatro niveles con criterios relacionados con el volumen, el tipo de diseño y la calidad de los estudios disponibles. Estos criterios se han adaptado de otros sistemas de jerarquización propuestos $^{33-37}$. Partiendo del nivel asignado en el sistema de clasificación previo, se propone un ajuste del nivel inicial siguiendo las recomendaciones de la propuesta GRADE ${ }^{38-41}$ :

- Disminuir el nivel de evidencia (principalmente en ensayos clínicos) si: 1) existen limitaciones importantes en la calidad de los estudios; 2) existen inconsistencias importantes entre estudios; 3) existen dudas sobre si la evidencia es directa o indirecta; 4) la información disponible es imprecisa o escasa; 5) es muy probable que los resultados tengan sesgos importantes.

- Aumentar el nivel de evidencia (principalmente en estudios obser- 
vacionales) si: 1) existen pruebas de una asociación muy fuerte (riesgo relativo $>50<0,2$ ) basadas en evidencia directa, sin amenazas importantes para la validez; 2) existen pruebas válidas de un gradiente dosis-respuesta; 3) se ha realizado un ajuste correcto de todos los factores de confusión posibles.

Se propone un sistema simplificado de graduación de las recomendaciones, adaptado de propuestas previas $^{2-6}$. Además del volumen y la calidad de la evidencia, a la hora de asignar el grado de recomendación se deben tener en cuenta otros criterios ${ }^{38-41}$ : la coherencia entre estudios, la posible influencia de factores de confusión o modificadores de efecto, la precisión de las estimaciones, la intensidad del efecto o la fuerza de la asociación, el grado de relación con la pregunta clínica (relación directa o indirecta), la aplicabilidad de la evidencia (efectividad y riesgo basal en la práctica clínica), el balance entre beneficios y riesgos o costes, la utilidad sobre los pacientes y sus preferencias y el potencial impacto de futuros estudios sobre la evidencia actual.

4. Finalmente, para los procedimientos diagnósticos y terapéuticos en los que se consideró necesario se establecieron las siguientes categorías de clasificación de la idoneidad:
- Opciones de primera elección (indicación rutinaria).

- Opciones de uso alternativo (indicación selectiva u opcional).

- Opciones inapropiadas o no recomendadas (innecesarias).

\section{Resultados}

Se realizaron un total de 61 preguntas en la Conferencia de Consenso, que se distribuyeron en: epidemiología y factores de riesgo (dos); perfil etiológico y patrones clínicos asociados (tres); pruebas diagnósticas (diez); factores pronósticos de gravedad (cuatro) y escalas de valoración de síntomas o gravedad (dos); riesgo de asma postbronquiolitis (una); tratamiento (28); prevención (ocho); y evaluación económica (tres).

En la tabla 1 se expone el resumen de las respuestas con las 61 preguntas planteadas en la Conferencia de Consenso, con su nivel de evidencia y grado de recomendación, según acuerdo llevado a cabo tras una reunión presencial de un día de trabajo de todo el Panel de Expertos y dos rondas de discusión por correo electrónico. Se llegó a consenso en 58 preguntas, y no fue posible en tres (las que corresponden a evaluación económica, centradas en el estudio coste-efectividad del palivizumab). 
Tabla 1. Resumen de las respuestas planteadas en la Conferencia de Consenso

\begin{tabular}{l|c|c}
\hline \multicolumn{1}{c|}{ Preguntas planteadas } & NE & GR \\
\hline \multicolumn{1}{c|}{ Epidemiología. Riesgo de bronquiolitis. Factores de riesgo } \\
\hline $\begin{array}{l}\text { E.1.1. ¿Cuál es el riesgo de BA en nuestro medio? } \\
\text { - Para población menor de dos años, la frecuencia de ingreso por BA se sitúa } \\
\text { en el 1-3,5\%,y para IRA de vías bajas por VRS en el 0,8-2,5\%. }\end{array}$ & II & \\
\hline $\begin{array}{l}\text { - Las estimaciones de frecuencia de consultas en Atención Primaria se sitúa } \\
\text { en el 4-20\%, y en urgencias en el 1-2\%. }\end{array}$ & III & \\
\hline $\begin{array}{l}\text { - Para población de riesgo, la frecuencia recogida en la mayoría de los estudios } \\
\text { se refiere a ingresos por IRA por VRS: } \leq 32 \text { SG en el 4,4-18\%; niños con DBP } \\
\text { en el 7,3-42\%, niños con CC en el 1,6-9,8\%. }\end{array}$ & II y III & \\
\hline
\end{tabular}

E.1.2. ¿Qué factores deben tenerse en cuenta para la estimación del riesgo de BA en nuestro medio?

- Los principales factores que se deben considerar en la estimación del riesgo de BA son: prematuridad, DBP, EPC, CC (fundamentalmente las complejas, hemodinámicamente inestables o con hiperflujo pulmonar), edad al inicio de la epidemia inferior a 3-6 meses. Otros factores son: hermanos mayores o asistencia a guardería, sexo masculino, exposición a tabaco (fundamentalmente durante la gestación), lactancia materna durante menos de 1-2 meses y variables asociadas a bajo nivel socioeconómico.

Perfil etiológico de la bronquiolitis aguda y patrones clínicos asociados

E.2.1. ¿Cuál es el perfil etiológico habitual en la BA?

- El VRS es el agente dominante de las BA, constituyendo el $56 \%$ de los casos ingresados por debajo de los dos años (intervalo $27 \%-73 \%$ ). Otros virus implicados, por orden descendente de frecuencia, son los rinovirus, adenovirus, metapneumovirus, virus de la gripe, parainfluenza, enterovirus y bocavirus.

- El porcentaje de BA con virus identificado en los trabajos en que se ha recurrido a técnicas de reacción en cadena de la polimerasa no supera el 75-80\%. Entre el $9 \%$ y el $27 \%$ de los casos presentan coinfección viral.

E.2.2. ¿Existe algún patrón clínico característico de las BA por VRS?

- La clínica no resulta útil para diferenciar la etiología de la BA. Aunque las BA por VRS tienen más sibilantes y trabajo respiratorio, mayor duración de síntomas y oxigenoterapia y se asocian a menor uso de antibióticos, estos datos están asociados a la menor edad de los pacientes que las presentan y no permiten predecir la etiología.

E.2.3. ¿La identificación etiológica es útil de cara al manejo terapéutico o el pronóstico de los pacientes con BA?

- En general, la identificación etiológico no resulta útil de cara al manejo de los pacientes con BA. No obstante, en lactantes pequeños ( $<3$ meses) con BA febriles en el medio hospitalario, la identificación de un VRS u otros virus respiratorios puede facilitar el manejo conservador de dichos pacientes y ahorrar intervenciones diagnósticas y/o terapéuticas.

Validez de las pruebas de diagnóstico rápido de infección por VRS

D.1.1. ¿Cuál es la muestra respiratoria más apropiada para la identificación del VRS en pacientes con BA?

\begin{tabular}{l|c|c}
$\begin{array}{l}\text { - Se recomienda la recogida de aspirados nasofaríngeos para la identificación } \\
\text { del VRS. }\end{array}$ & II & B \\
\hline
\end{tabular}




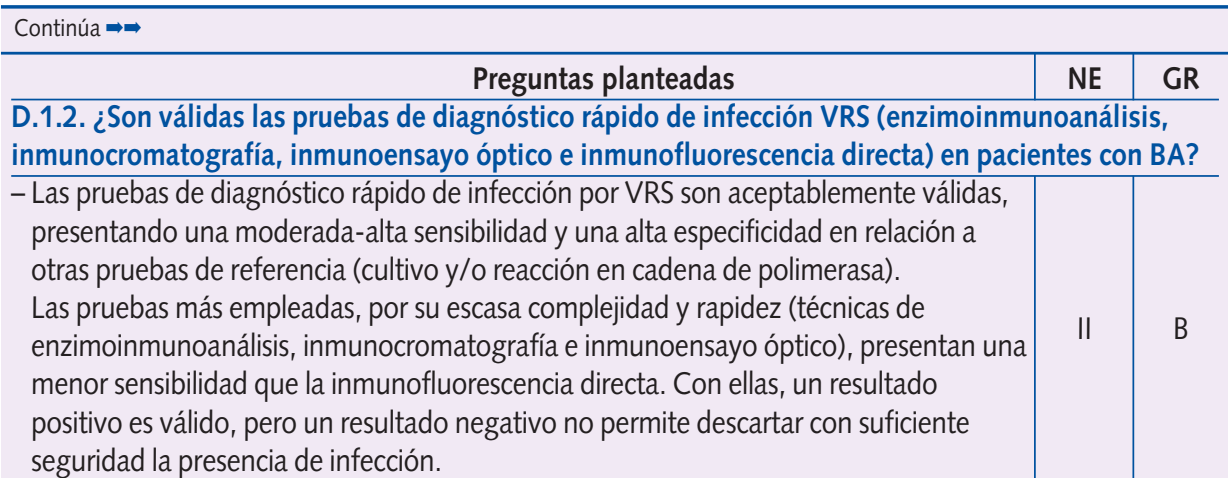

D.1.3. ¿Se recomienda el empleo rutinario de las pruebas de diagnóstico rápido de infección VRS en los pacientes con $\mathrm{BA}$ ?

- No se recomienda el empleo rutinario de pruebas de diagnóstico rápido de infección por VRS en los pacientes con BA.

D.1.4. ¿Debe considerarse en el empleo de las técnicas de diagnóstico rápido algún criterio relacionado con la edad, ámbito de asistencia del paciente (cuidados intensivos, hospitalización, urgencias, primaria), antecedentes o gravedad?

- Podría considerarse el uso de pruebas de diagnóstico rápido de VRS en el medio hospitalario con pacientes menores de tres meses. Aunque los resultados negativos de estas pruebas no permiten descartar la presencia de infección por VRS y por lo tanto evitar el aislamiento de los pacientes, en el curso de epidemias, un resultado positivo facilitaría la agrupación de enfermos hospitalizados.

Pruebas diagnósticas en el manejo de la bronquiolitis aguda: radiografía de tórax, pulsioximetría y pruebas de cribado de infección bacteriana

D.2.1. ¿Resulta útil la realización de una radiografía de tórax para el manejo de la BA?

- No se recomienda el uso rutinario de la radiografía de tórax en la BA.

D.2.2. ¿Existe algún criterio que permita identificar a los pacientes en los que la radiografía de tórax sea útil?

- No existen signos o síntomas concretos que permitan identificar a los pacientes que se beneficiarán de la realización de una radiografía de tórax. No obstante podría considerarse en pacientes con BA que presentan un deterioro clínico evidente o en los que existen dudas diagnósticas.

D.2.3. ¿Resulta útil la medición de la saturación de oxígeno en los pacientes con BA?

- La medición de la saturación de oxígeno resulta útil en la valoración inicial o en el control de los cambios clínicos de los pacientes con BA. Sin embargo, no parece justificada la monitorización rutinaria mantenida.

D.2.4. ¿Existe algún criterio que permita identificar a los pacientes que precisan la medición de la saturación de oxígeno?

- Se recomienda la medición de la $\mathrm{SatO}_{2}$ en la valoración inicial de todos los pacientes y en el control de cambios clínicos en los pacientes con compromiso respiratorio. III y IV C D.2.5. ¿Qué puntos de corte de la saturación de oxígeno orientan en el manejo del paciente con BA? - Los pacientes con $\mathrm{SatO}_{2}>94 \%$ y sin otros signos o síntomas de compromiso respiratorio o afectación general pueden ser manejados habitualmente a nivel ambulatorio 


\begin{tabular}{|c|c|c|}
\hline \multicolumn{3}{|l|}{ Continúa $\Rightarrow$} \\
\hline $\begin{array}{r}\text { Preguntas planteadas } \\
\end{array}$ & NE & GR \\
\hline - Los pacientes con $\mathrm{SatO}_{2}<92 \%$ requieren oxigenoterapia. & III & C \\
\hline $\begin{array}{l}\text { - En pacientes con } \mathrm{SatO}_{2} \text { entre el } 92 \% \text { y el } 94 \% \text {, se valorará individualmente } \\
\text { la administración de oxígeno y el manejo ambulatorio, considerando conjuntamente } \\
\text { otros parámetros: trabajo respiratorio, alimentación, ambiente familiar, posibilidad } \\
\text { de control, etc. }\end{array}$ & IV & C \\
\hline \multicolumn{3}{|l|}{$\begin{array}{l}\text { D.2.6. ¿Existe algún criterio que permita identificar a los pacientes con BA que requieren la } \\
\text { realización de cribado de infección bacteriana? }\end{array}$} \\
\hline $\begin{array}{l}\text { - No existen criterios clínicos con suficiente capacidad predictiva como para seleccionar } \\
\text { los casos que se beneficiarían de dichas pruebas; podría considerarse su realización } \\
\text { en pacientes con fiebre persistente, edad inferior a un mes o con signos o síntomas } \\
\text { de gravedad. }\end{array}$ & III y IV & C \\
\hline \multicolumn{3}{|l|}{ Factores pronósticos de gravedad en bronquiolitis agudas } \\
\hline \multicolumn{3}{|l|}{ PN.1.1. ¿Qué factores de riesgo se asocian con mayor gravedad? } \\
\hline $\begin{array}{l}\text { - En pacientes ingresados con BA son factores de riesgo documentados de estancias } \\
\text { prolongadas o ingreso en UCIP: la displasia broncopulmonar y/o enfermedad } \\
\text { pulmonar crónica, la prematuridad, las cardiopatías congénitas y la edad menor } \\
\text { de tres meses. }\end{array}$ & II y IV & B \\
\hline $\begin{array}{l}\text { - Son marcadores de gravedad asociados a la indicación de ingreso en pacientes } \\
\text { atendidos en urgencias y/o a la duración de la estancia hospitalaria en pacientes } \\
\text { ingresados: el aspecto tóxico, la taquipnea, la hipoxia }\left(\mathrm{SatO}_{2}<92 \%\right) \text {, la presencia } \\
\text { de atelectasia o infiltrado en la radiografía de tórax, el trabajo respiratorio } \\
\text { aumentado, los signos de deshidratación, la taquicardia y la fiebre. }\end{array}$ & III & B \\
\hline \multicolumn{3}{|l|}{ PN.1.2. ¿Existe algún modelo predictivo válido de gravedad? } \\
\hline $\begin{array}{l}\text { - Aunque existen diversos modelos predictivos, ninguno ha mostrado suficiente } \\
\text { validez como para recomendar su uso en la práctica clínica. }\end{array}$ & II & B \\
\hline \multicolumn{3}{|l|}{$\begin{array}{l}\text { PN.1.3. ¿Qué factores de riesgo o marcadores clínicos de gravedad deben considerarse en la } \\
\text { decisión de ingreso hospitalario? }\end{array}$} \\
\hline $\begin{array}{l}\text { - En la decisión de ingreso hospitalario deben considerarse los siguientes factores de } \\
\text { riesgo: prematuridad, DBP y/o EPC, CC y edad menor de tres meses. Así mismo se } \\
\text { deben tener en cuenta los marcadores de gravedad: presencia de apneas, aspecto } \\
\text { tóxico, hipoxia (cianosis, SatO }{ }_{2} \text { inferior a } 92 \% \text { ), taquipnea (según edad), rechazo } \\
\text { de tomas o problemas de hidratación. Además, debe valorarse la capacidad } \\
\text { del entorno familiar para realizar los cuidados que requiere el paciente con BA. }\end{array}$ & III y IV & C \\
\hline \multicolumn{3}{|l|}{$\begin{array}{l}\text { PN.1.4. ¿Qué factores de riesgo o marcadores clínicos de gravedad deben ser considerados } \\
\text { en la decisión de ingreso en UCI? }\end{array}$} \\
\hline $\begin{array}{l}\text { - No existen criterios específicos de ingreso en UCIP aplicables a pacientes con BA, } \\
\text { por lo que les serían aplicables los criterios generales. }\end{array}$ & IV & $\mathrm{D}$ \\
\hline \multicolumn{3}{|l|}{ Escalas de valoración de síntomas o gravedad } \\
\hline \multicolumn{3}{|l|}{$\begin{array}{l}\text { PN.2.1. ¿Existe alguna escala más válida y precisa para la valoración de síntomas o gravedad } \\
\text { de BA? }\end{array}$} \\
\hline $\begin{array}{l}\text { - Ninguna escala de valoración de síntomas o gravedad de BA ha demostrado } \\
\text { ser más válida o precisa como para recomendar su aplicación preferente } \\
\text { en la práctica clínica. }\end{array}$ & III & $C$ \\
\hline & & \\
\hline
\end{tabular}




\begin{tabular}{|c|c|c|}
\hline \multicolumn{3}{|l|}{ Continúa $\Rightarrow$} \\
\hline $\begin{array}{c}\text { Preguntas planteadas } \\
\end{array}$ & NE & GR \\
\hline \multicolumn{3}{|l|}{ PN.2.2. ¿Qué síntomas o signos deben incluirse en la valoración de la gravedad de la BA? } \\
\hline $\begin{array}{l}\text { - Los principales síntomas o signos que deben considerarse en la valoración de la } \\
\text { gravedad de la BA son la hipoxia, la intensidad y extensión de sibilantes y retracciones } \\
\text { torácicas y la FR. Otros signos o síntomas a considerar son la FC, el nivel de conciencia, } \\
\text { el grado de hidratación, la presencia de apneas y otros signos directos o indirectos } \\
\text { de compromiso respiratorio (rechazo de tomas, tos, capacidad de vocalización, grado } \\
\text { de ventilación). }\end{array}$ & II y III & B \\
\hline $\begin{array}{l}\text { - Toda valoración de síntomas o signos de gravedad debe realizarse tras una adecuada } \\
\text { aspiración secreciones nasofaríngeas, para mejorar la precisión de las mediciones. }\end{array}$ & IV & D \\
\hline \multicolumn{3}{|l|}{ Riesgo de asma postbronquiolitis } \\
\hline \multicolumn{3}{|l|}{ P.3.1. ¿Es la BA un factor de riesgo independiente de asma? } \\
\hline $\begin{array}{l}\text { - Parece existir una consistente y fuerte asociación entre ingreso por BA o IRA baja } \\
\text { antes de los dos años y episodios de sibilantes recurrentes en los primeros cinco } \\
\text { años de vida. Sin embargo no está claro si en años posteriores dicha asociación } \\
\text { se prolonga, existiendo información discordante sobre la asociación entre ingreso } \\
\text { por BA o IRA baja y asma. }\end{array}$ & II & B \\
\hline
\end{tabular}

\section{Medidas de soporte}

Tratamiento de la bronquiolitis aguda

T.1.1. ¿Debe hacerse alguna recomendación específica sobre el uso de tratamiento de soporte (fluidoterapia, suplementos de oxígeno, desobstrucción nasal, posición) en el manejo de la BA?

No existe un tratamiento de soporte específico en el manejo de la BA. Se consideran recomendaciones basadas en la opinión de expertos y en la buena práctica clínica las siguientes:

\begin{tabular}{|c|c|c|}
\hline $\begin{array}{l}\text { - Los niños con dificultad respiratoria grave y/o cianosis y/o } \mathrm{SatO}_{2}<92 \% \\
\text { deben recibir } \mathrm{O}_{2} \text { suplementario. }\end{array}$ & $\|-2$ & C \\
\hline $\begin{array}{l}\text { - Se recomienda aspirar las secreciones respiratorias antes de las tomas, antes de } \\
\text { valorar la gravedad, antes de cada tratamiento inhalado y cuando se objetiven } \\
\text { signos de obstrucción de vías altas. }\end{array}$ & III y IV & C \\
\hline - Se recomienda aplicar medidas posturales: elevación de la cabecera de la cuna. & III y IV & C \\
\hline - Se debe valorar el estado de hidratación y la capacidad de tomar líquidos. & III y IV & C \\
\hline $\begin{array}{l}\text { - Se recomienda fraccionar y/o espesar las tomas si se objetiva dificultad para } \\
\text { la ingesta. }\end{array}$ & III y IV & C \\
\hline $\begin{array}{l}\text { - La alimentación por SNG puede ser una opción en los niños con riesgo } \\
\text { de deshidratación y desnutrición. }\end{array}$ & III y IV & C \\
\hline $\begin{array}{l}\text { - En los niños más graves o en los que no toleren la vía oral se recomienda canalizar } \\
\text { una vía e hidratar vía IV. }\end{array}$ & II-2 & C \\
\hline - Evitar exposición al tabaco. & III y IV & C \\
\hline \multicolumn{3}{|l|}{ Broncodilatadores } \\
\hline \multicolumn{3}{|l|}{ T.2.a.1. ¿Es eficaz el salbutamol inhalado en el tratamiento de la BA? } \\
\hline $\begin{array}{l}\text { - No se recomienda el uso rutinario de salbutamol en el tratamiento de la BA, } \\
\text { por tener una relación beneficio-riesgo desfavorable. }\end{array}$ & | y ||-1 & A \\
\hline
\end{tabular}

T.2.a.2. ¿Es eficaz el salbutamol oral en el tratamiento de la BA?

No debe emplearse el salbutamol oral en el tratamiento de la BA, por tener con una relación beneficio-riesgo muy desfavorable. 
Continúa $\Rightarrow$

\section{Preguntas planteadas}

NE $\quad$ GR

T.2.a.3. ¿Es útil la realización de una prueba terapéutica con salbutamol inhalado de forma sistemática en la BA?

- El ensayo terapéutico con salbutamol inhalado es una opción alternativa en BA de intensidad moderada-grave (especialmente en los mayores de seis meses y/o con historia personal/familiar de atopia). En cualquier caso, si se decide realizar un ensayo terapéutico, este debería ser suspendido si tras su aplicación no se objetiva mejoría clínica.

T.2.b.1. ¿Es eficaz la adrenalina nebulizada en el tratamiento de la BA?

- No se recomienda el uso rutinario de adrenalina nebulizada en el tratamiento de la BA, por tener una relación beneficio-riesgo parcialmente desfavorable.

- Por su perfil terapéutico y de seguridad sería el broncodilatador preferido en urgencias y en pacientes hospitalizados.

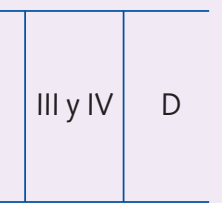

T.2.b.2. ¿Es eficaz la adrenalina subcutánea en el tratamiento de la BA?

- La adrenalina subcutánea no es eficaz en la BA.

\begin{tabular}{c|c}
\hline Iy II-1 & $A$ \\
\hline IV & $D$ \\
\hline
\end{tabular}

T.2.b.3. ¿Es útil la realización de una prueba terapéutica con adrenalina nebulizada de forma sistemática en la BA?

- El ensayo terapéutico con adrenalina nebulizada es una opción alternativa en BA de intensidad moderada-grave (especialmente en los menores de seis meses) y en pacientes hospitalizados o atendidos en urgencias o en centros de salud, si hay posibilidad de observación. En cualquier caso, si se decide realizar un ensayo terapéutico, este debería ser suspendido si tras su aplicación no se objetiva mejoría clínica.

T.2.b.4. ¿Es más eficaz la adrenalina que el salbutamol?

- La adrenalina constituye el agente broncodilatador de primera elección en el tratamiento de la BA por su relación entre riesgos, beneficios y costes.

T.2.c.1. ¿Es eficaz el bromuro de ipratropio nebulizado en el tratamiento de la BA?

- El bromuro de ipratropio no es eficaz en la BA.

Corticoides

T.3.1. ¿Son eficaces los corticoides inhalados en el tratamiento de la BA?

- Los corticoides inhalados no son útiles en el tratamiento de la BA por tener una relación beneficio-riesgo desfavorable.

T.3.2. ¿Son eficaces los corticoides sistémicos en el tratamiento de la BA?

- Los corticoides sistémicos no parecen útiles en el tratamiento de la BA, aunque presentan equilibrio entre riesgos (potenciales, aunque poco constatados por el escaso tiempo de aplicación) y beneficios (diferencias significativas en niños atendidos en urgencias con corticoides orales durante 3-5 días, principalmente), por lo que no podría considerarse inadecuado su uso restringido.

Ribavirina

T.4.1. ¿Es eficaz la ribavirina nebulizada en el tratamiento sistemático de la BA por VRS?

- No se debe considerar su uso sistemático, dado el predominio de los riesgos (problemas de administración, efectos secundarios y teratogenicidad) sobre los beneficios (cierta mejoría en algunas variables, no consistente entre los estudios y marginal en la mayoría de los casos), sin perder en cuenta los costes económicos de su administración. 


\begin{tabular}{|c|c|c|}
\hline Preguntas planteadas & NE & GR \\
\hline \multicolumn{3}{|l|}{ T.4.2. ¿Es más útil la ribavirina en algún grupo de pacientes? (relación riesgo-beneficio) } \\
\hline $\begin{array}{l}\text { - El efecto de la ribavirina tiene un escaso impacto clínico; no obstante, podría } \\
\text { considerarse su uso restringido en pacientes con infecciones respiratorias por VRS } \\
\text { graves de alto riesgo. }\end{array}$ & IV & $\mathrm{D}$ \\
\hline \multicolumn{3}{|l|}{ Antibióticos } \\
\hline \multicolumn{3}{|l|}{ T.5.1. ¿Son eficaces los antibióticos en el tratamiento de la BA? } \\
\hline $\begin{array}{l}\text { - Los antibióticos no son eficaces en la BA y su uso se considera inapropiado, por el claro } \\
\text { predominio de riesgos (favoreciendo la exposición innecesaria y sus consecuencias } \\
\text { sobre resistencias bacterianas, efectos adversos y costes) sobre beneficios (posibilidad } \\
\text { de tratar pacientes con infección bacteriana, pero son infrecuentes y no graves } \\
\text { en su mayoría). }\end{array}$ & $\|-1$ & B \\
\hline \multicolumn{3}{|l|}{ Suero salino hipertónico } \\
\hline \multicolumn{3}{|l|}{ T.6.1. ¿Es eficaz el suero salino hipertónico nebulizado en el tratamiento de la BA? } \\
\hline $\begin{array}{l}\text { - El suero salino hipertónico es eficaz y útil en el manejo de la BA del niño } \\
\text { hospitalizado, por tener una relación favorable entre riesgos (no constatados), } \\
\text { beneficios (en días de hospitalización, mejoría clínica en los tres primeros días } \\
\text { y potencial disminución de la hospitalización) y costes (prácticamente nulo, salvo } \\
\text { la adquisición del nebulizador). Se plantea la duda de que la pauta utilizada } \\
\text { ( } 2 \text { a } 4 \text { ml con dosis repetidas cada ocho horas -o más frecuentemente-y durante } \\
\text { cinco días) sea factible en el medio ambulatorio (urgencias o domicilio). }\end{array}$ & I & A \\
\hline
\end{tabular}

T.6.2. ¿Debe usarse asociado a broncodilatadores?

- De momento los estudios publicados abogan por el uso simultáneo de las nebulizaciones de suero salino hipertónico con broncodilatadores

(beta-2-adrenérgicos o adrenalina), si bien no hay datos que sugieran sinergia; caben dos consideraciones simultáneas: la aplicación actual de suero salino hipertónico con broncodilatadores tendría el teórico beneficio de evitar el potencial broncoespasmo de una solución hipertónica en el árbol bronquial; si se considera la utilización de broncodilatadores en el manejo de la BA, considerar vehiculizarlos mejor con salino hipertónico que con salino fisiológico.

Inmunoglobulinas

T.7.1. ¿Es eficaz la inmunoglobulina IV en el tratamiento de la BA?

- La inmunoglobulina intravenosa no es eficaz en el tratamiento de la BA, presentando un balance desfavorable entre riesgos (potenciales), beneficios (no constados) y costes (elevados).

T.7.2. ¿Es eficaz la inmunoglobulina aerosolizada en el tratamiento?

- La inmunoglobulina aerosolizada no es eficaz en el tratamiento de la BA. Medidas de soporte respiratorio

T.8.1. ¿Debe hacerse alguna recomendación específica sobre el tipo de soporte respiratorio en la BA?

- El soporte respiratorio de la BA no requiere recomendaciones específicas respecto a otras entidades con dificultad respiratoria, salvo quizás el predominio en esta entidad del fracaso respiratorio hipercápnico y la utilidad de la ventilación no invasiva. 


\begin{tabular}{|c|c|c|}
\hline \multicolumn{3}{|l|}{ Continúa $\Rightarrow$} \\
\hline Preguntas planteadas & NE & GR \\
\hline $\begin{array}{l}\text { - En la CPAP el balance entre riesgos (método de ventilación no invasiva, } \\
\text { sin efectos adversos constatados) y beneficios (mejoría clínica y } \\
\text { gasométrica a corto plazo, pero sin correlato con otras medidas de impacto clínico, } \\
\text { como ingreso) puede ser favorable en las BA que presentan dificultad respiratoria } \\
\text { moderada-grave o apnea recurrente, especialmente por la posibilidad de evitar } \\
\text { intubación y ventilación mecánica de estos pacientes. }\end{array}$ & $\begin{array}{l}\|-2 \\
y \text { III }\end{array}$ & B \\
\hline \multicolumn{3}{|l|}{$\begin{array}{l}\text { T.8.2. ¿Existen criterios clínicos que delimiten los pacientes que requieren un aumento } \\
\text { del soporte respiratorio (CPAP, ventilación con presión positiva intermitente, ventilación } \\
\text { de alta frecuencia y oxigenación con membrana extracorpórea)? }\end{array}$} \\
\hline $\begin{array}{l}\text { - En la BA son aplicables los criterios habituales de manejo de pacientes con } \\
\text { dificultad respiratoria y las distintas modalidades de ventilación mecánica. }\end{array}$ & III y IV & $\mathrm{D}$ \\
\hline $\begin{array}{l}\text { - Se ha propuesto la ventilación no invasiva (CPAP o BiPAP) como una alternativa o } \\
\text { paso intermedio, antes de la ventilación invasiva, de forma particular en aquellos } \\
\text { pacientes con crisis de apnea frecuentes. }\end{array}$ & $\|-2$ y $\|$ & B \\
\hline \multicolumn{3}{|l|}{ T.8.3. ¿Es eficaz el heliox en el tratamiento de la BA? } \\
\hline $\begin{array}{l}\text { - Se debe considerar el uso selectivo del heliox en BA moderada-grave } \\
\text { utilizado con mascarilla con reservorio y de forma continua con } \\
\text { un concentración 70/30; en los casos de BA grave y/o refractarios } \\
\text { al tratamiento con heliox a través de mascarilla con reservorio se recomienda } \\
\text { utilizar el heliox combinado con CPAP. }\end{array}$ & $\|-1$ y $\|$ & B \\
\hline \multicolumn{3}{|l|}{ T.8.4. ¿Es eficaz el surfactante en el tratamiento de la BA? } \\
\hline $\begin{array}{l}\text { - Se puede considerar el uso selectivo del surfactante en los casos de BA } \\
\text { grave que precisen ventilación mecánica, dada la favorable relación } \\
\text { entre beneficios (menor días de ingreso en cuidados intensivos y necesidad } \\
\text { de ventilación mecánica) y riesgos (nulo). Aunque no hay estudios de costes } \\
\text { al respecto, es preciso tener en cuenta que las preparaciones de surfactante } \\
\text { son caras. }\end{array}$ & $\|-1$ & B \\
\hline \multicolumn{3}{|l|}{ T.8.5. ¿Son eficaces las metilxantinas en el tratamiento de la BA? } \\
\hline - No se recomienda el uso general de las metilxantinas en el tratamiento de la BA. & IV & $\mathrm{D}$ \\
\hline $\begin{array}{l}\text { - Se podrían considerar como de uso alternativo en la BA con apnea en neonatos } \\
\text { y/o en lactantes con antecedentes de prematuridad. }\end{array}$ & III & C \\
\hline \multicolumn{3}{|l|}{ T.8.6. ¿Es eficaz el óxido nítrico en el tratamiento de la BA? } \\
\hline - El óxido nítrico no es eficaz en el tratamiento de la BA. & IV & $\mathrm{D}$ \\
\hline \multicolumn{3}{|l|}{ Fisioterapia } \\
\hline \multicolumn{3}{|l|}{ T.9.1. ¿Es eficaz la fisioterapia en el tratamiento de la BA } \\
\hline - No se recomienda el uso de fisioterapia respiratoria en el tratamiento de la BA. & II-1 & B \\
\hline \multicolumn{3}{|l|}{ Otras intervenciones } \\
\hline \multicolumn{3}{|l|}{ T.10.1. ¿Es eficaz la DNA-asa recombinante humana nebulizada en el tratamiento de la BA? } \\
\hline - La DNA-asa recombinante humana nebulizada no es eficaz en el tratamiento de la BA. & 1 & A \\
\hline \multicolumn{3}{|l|}{ T.10.2. ¿Es eficaz el interferón IM en el tratamiento de la BA? } \\
\hline -El interferón IM no es eficaz en el tratamiento de la BA. & II-1 & B \\
\hline \multicolumn{3}{|l|}{ T.10.3. ¿Es eficaz la furosemida nebulizada en el tratamiento de la BA? } \\
\hline - La furosemida nebulizada no es eficaz en el tratamiento de la BA. & II-1 & B \\
\hline
\end{tabular}




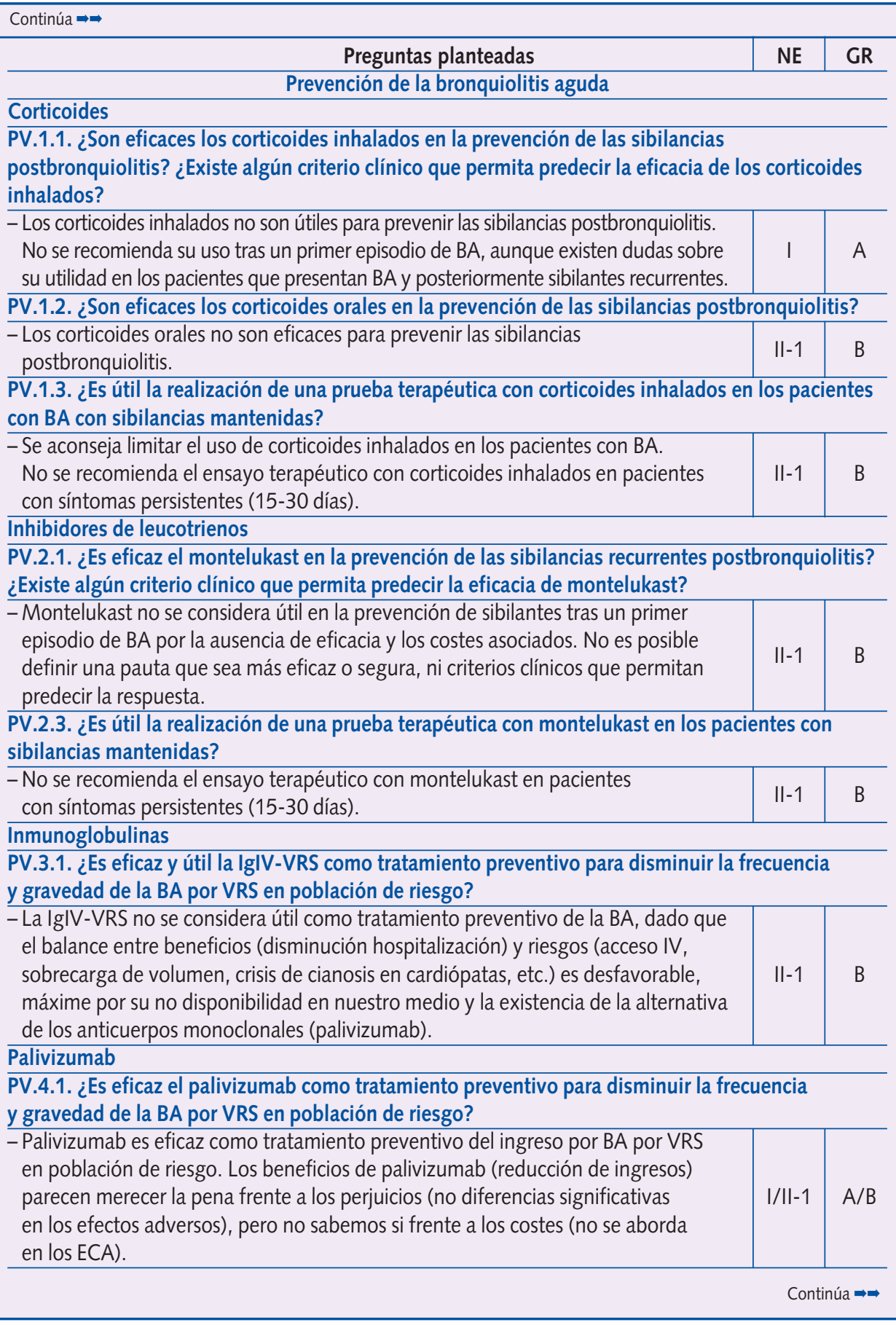




\begin{tabular}{|c|c|c|}
\hline \multicolumn{3}{|l|}{ Continúa $\Rightarrow$} \\
\hline Preguntas planteadas & NE & GR \\
\hline \multicolumn{3}{|c|}{$\begin{array}{l}\text { PV.4.2. ¿Es efectivo (en estudios observacionales) el palivizumab como tratamiento preventivo } \\
\text { para disminuir la frecuencia y gravedad? }\end{array}$} \\
\hline $\begin{array}{l}\text { - La efectividad de palivizumab en estudios observacionales parece similar a la eficacia } \\
\text { observada en los ensayos clínicos originales, considerando que si es efectivo respecto } \\
\text { a la hospitalización (aunque con una gran heterogeneidad entre países), pero no } \\
\text { respecto a la frecuencia y gravedad (ingresos en UCI, necesidad de VM y mortalidad) } \\
\text { de la BA. }\end{array}$ & $\| 2 / I I I$ & $\mathrm{~B} / \mathrm{C}$ \\
\hline $\begin{array}{l}\text { Evaluación económica de la bronquiolitis aguda } \\
\text { C.1. ¿En qué grupo de niños prematuros, por edad gestacional, patología asociada y } \\
\text { postconcepcional, podemos considerar coste-efectiva la inmunoprofilaxis con palivi } \\
\text { en nuestro medio? }\end{array}$ & & \\
\hline $\begin{array}{l}\text { - Solo aceptando la asunción de que palivizumab reduce la mortalidad y el riesgo } \\
\text { de asma, este tratamiento resultaría coste-efectivo en los grupos de mayor riesgo. } \\
\text { No se ha alcanzado un consenso sobre los grupos de riesgo en los que palivizumab } \\
\text { podría ser coste-efectivo. Las opciones propuestas varían desde la más restrictiva, } \\
\text { limitada a prematuros de } 23-32 \text { SG con EPC, o sin EPC con múltiples factores } \\
\text { de riesgo o con EPC grave, a la más amplia (vinculada a la aceptación de todas } \\
\text { las asunciones) que contemplaría prematuros } \leq 32 \text { SG (con } \leq 6 \text { meses de edad) } \\
\text { o } \leq 28 \text { SG (con } \leq 12 \text { meses de edad) o con EPC activa (con } \leq 2 \text { años de edad) } \\
\text { y prematuros de } 32-35 \text { SG (con } \leq 6 \text { meses de edad) con dos factores de riesgo: } \\
\text { edad < } 3 \text { meses en la estación y hermanos y/o guardería. No existe consenso } \\
\text { sobre el nivel de evidencia que apoya estas recomendaciones, habiéndose } \\
\text { propuesto niveles I y II. }\end{array}$ & $\begin{array}{c}\text { No se } \\
\text { establece } \\
\text { nivel de } \\
\text { evidencia }\end{array}$ & \\
\hline
\end{tabular}

C.2. ¿En qué grupo de niños con cardiopatías congénitas, por tipo de cardiopatía y edad postconcepcional, podemos considerar coste-efectiva la inmunoprofilaxis con palivizumab en nuestro medio?

\begin{tabular}{l|c|c}
\hline - No se ha alcanzado un consenso sobre el grupo de niños con cardiopatía congénita & \\
en los que la inmunoprofilaxis con palivizumab podría ser coste-efectiva. No obstante & No se \\
se ha considerado que su uso podría ser eficiente en < 2 años con cardiopatía & establece \\
congénita (no corregida o con intervención paliativa) con alteración hemodinámica & nivel de \\
significativa, en tratamiento con insuficiencia cardiaca, hipertensión pulmonar & eviden \\
moderada o cardiopatías cianógenas. & & \\
\hline
\end{tabular}

C.3. ¿Son aceptables las asunciones que se han empleado en los modelos económicos de coste efectividad de palivizumab?

- Existen dudas sobre la validez de las asunciones de que la inmunoprofilaxis con palivizumab reduce la mortalidad o el riesgo de asma, ya que no se sustentan en los resultados de ensayos clínicos, sino que son extrapolaciones a partir de estudios observacionales sobre riesgos asociados a ingreso por infección por VRS.

No se
establece
nivel de
evidencia

BA: bronquiolitis aguda; BIPAP: presión positiva con dos niveles de presión; CPAP: presión positiva continua en la vía respiratoria; CC: cardiopatías congénitas; DBP: displasia broncopulmonar; ECA: ensayo clínico aleatorizado; EPC: enfermedad pulmonar crónica; FC: frecuencia cardiaca; FR: frecuencia respiratoria; GR: grado de recomendación; IM: intramuscular; IRA: infección respiratoria aguda; NE: nivel de evidencia; $\mathrm{SatO}_{2}$ : saturación de oxígeno; SG: semanas de gestación; UCIP: unidad de cuidados intensivos pediátricos; VM: ventilación mecánica; VRS: virus respiratorio sincitial. 
Más de la mitad de las preguntas al Panel de Expertos versan sobre tratamiento y prevención, subdivididas en los siguientes temas:

A. Tratamiento: medidas de soporte (una), broncodilatadores (ocho), corticoides (dos), ribavirina (dos), antibióticos (una), suero salino hipertónico (dos), inmunoglobulinas (dos), medidas de soporte respiratorio (seis), fisioterapia (una) y otras intervenciones (tres).

B. Prevención: corticoides (tres), inhibidores de leucotrienos (dos), inmunoglobulinas (una) y palivizumab (dos).

En la tabla 2 se expone la clasificación de la idoneidad (primera elección o uso sistemático; uso alternativo u opcional; uso inapropiado o innecesario) de las distintas intervenciones terapéuticas y preventivas en la BA, sobre la base del análisis de la revisión sistemática, nivel de evidencia y grado de recomendación que se exponen en la tabla $1^{1}$.

\section{Discusión}

La BA es un ejemplo paradigmático de inadecuación de la práctica clínica a la evidencia científica en el campo del tratamiento. El tratamiento de la BA varía en cada brote epidémico anual dependiendo, en muchos casos, del artículo de "moda". Según esto, no es infrecuente que un Residente de Pediatría en forma- ción pueda haber tratado a estos pacientes un año preferentemente con adrenalina, otro con salbutamol y un tercero con suero salino hipertónico.

Variabilidad injustificada, sin duda. La cuestión está en definir el medicamento correcto para el paciente adecuado en la dosis apropiada y durante el tiempo oportuno ${ }^{42}$. La existencia de múltiples ECA en los últimos 25 años sobre las potenciales intervenciones farmacológicas en la BA (principalmente broncodilatadores, antiinflamatorios y antivíricos) y el escaso avance en el manejo de los infrecuentes casos de BA moderada-grave llegan a plantear incluso la duda de si es necesario continuar algunas líneas de investigación. El mayor avance en el desarrollo farmacológico de la BA en los últimos años se debe al campo de la prevención (desarrollo de anticuerpos monoclonales como inmunoprofilaxis), mientras que en el tratamiento son menos las novedades (heliox, suero salino hipertónico, etc.). Los objetivos del tratamiento de la BA son: disminuir la morbimortalidad, disminuir los costes sociales y económicos (reducir los ingresos hospitalarios y días de hospitalización) y disminuir las secuelas a largo plazo (patología respiratoria recurrente y, quizás, asma).

Según los resultados de la tabla 2 , conviene plantear algunos comentarios: 
Tabla 2. Clasificación de la idoneidad de procedimientos terapéuticos y preventivos en la bronquiolitis aguda

\begin{tabular}{|c|c|c|c|}
\hline Procedimiento & $\begin{array}{l}\text { Primera elección } \\
\text { o uso sistemático }\end{array}$ & Uso alternativo u opcional & $\begin{array}{l}\text { Uso inapropiado } \\
\text { o innecesario }\end{array}$ \\
\hline \multicolumn{4}{|l|}{ Tratamiento } \\
\hline $\begin{array}{l}\text { Suplementos } \\
\text { de oxígeno }\end{array}$ & $\begin{array}{l}\text { Saturación }<92 \% 0 \\
\text { dificultad respiratoria } \\
\text { grave }\end{array}$ & $\begin{array}{l}\text { Saturación entre } 92 \text { y } 94 \% \\
\text { (valorar otros parámetros) }\end{array}$ & $\begin{array}{l}\text { Saturación }>94 \% \\
\text { sin compromiso } \\
\text { respiratorio }\end{array}$ \\
\hline Salbutamol oral & & & Uso inapropiado \\
\hline Salbutamol inhalado & & $\begin{array}{l}\text { Ensayo terapéutico en BA } \\
\text { moderada-grave } \\
\text { (principalmente si > } 6 \text { meses); } \\
\text { mantener si mejoría clínica }\end{array}$ & $\begin{array}{l}\text { No indicado de forma } \\
\text { rutinaria }\end{array}$ \\
\hline Adrenalina nebulizada & & $\begin{array}{l}\text { Ensayo terapéutico en BA } \\
\text { moderada-grave }<6 \text { meses } \\
\text { ingresados o de urgencias } \\
\text { (en centros de salud si hay } \\
\text { opción de observación); } \\
\text { mantener si mejoría clínica }\end{array}$ & $\begin{array}{l}\text { No indicada de forma } \\
\text { rutinaria }\end{array}$ \\
\hline Adrenalina subcutánea & & & No recomendada \\
\hline Bromuro de ipatropio & & & Uso inapropiado \\
\hline Corticoides inhalados & & & Uso inapropiado \\
\hline Corticoides sistémicos & & $\begin{array}{l}\text { No se recomienda, pero no se } \\
\text { consideraría inapropiado en } \\
\text { BA moderada-grave asociados } \\
\text { a broncodilatadores }\end{array}$ & No recomendado \\
\hline Ribavirina nebulizada & & $\begin{array}{l}\text { Pacientes graves de alto riesgo } \\
\text { (UCl) con infección por VRS }\end{array}$ & $\begin{array}{l}\text { No indicado de forma } \\
\text { rutinaria }\end{array}$ \\
\hline Antibióticos & & & Uso inapropiado \\
\hline $\begin{array}{l}\text { Suero salino } \\
\text { hipertónico nebulizado }\end{array}$ & & $\begin{array}{l}\text { Ensayo terapéutico } \\
\text { junto con broncodilatadores }\end{array}$ & $\begin{array}{l}\text { Uso inapropiado } \\
\text { en BA leves }\end{array}$ \\
\hline Inmunoglobulina IV & & & Uso inapropiado \\
\hline $\begin{array}{l}\text { Inmunoglobulina } \\
\text { aerosolizada }\end{array}$ & & & Uso inapropiado \\
\hline Heliox & $\begin{array}{l}\text { BA grave }(\mathrm{UCl}) \\
\text { con compromiso } \\
\text { respiratorio }\end{array}$ & $\begin{array}{l}\text { BA moderada-grave con } \\
\text { compromiso respiratorio } \\
\text { creciente }\end{array}$ & $\begin{array}{l}\text { No indicado de forma } \\
\text { rutinaria }\end{array}$ \\
\hline Surfactante & & $\mathrm{BA}$ grave en $\mathrm{UCl}$ & $\begin{array}{l}\text { No indicado de forma } \\
\text { rutinaria }\end{array}$ \\
\hline Metilxantinas & & $\begin{array}{l}\text { BA con apnea en neonatos } \\
\text { y/o lactantes con } \\
\text { antecedente de prematuridad }\end{array}$ & $\begin{array}{l}\text { Uso inapropiado } \\
\text { en otros escenarios } \\
\text { clínicos }\end{array}$ \\
\hline Oxido nítrico & & & Uso inapropiado \\
\hline $\begin{array}{l}\text { Fisioterapia } \\
\text { respiratoria }\end{array}$ & & & Uso inapropiado \\
\hline & & & Continúa $\Rightarrow$ \\
\hline
\end{tabular}




\begin{tabular}{|c|c|c|c|}
\hline \multicolumn{4}{|l|}{ Continúa $\Rightarrow$} \\
\hline Procedimiento & $\begin{array}{l}\text { Primera elección } \\
\text { o uso sistemático }\end{array}$ & Uso alternativo u opcional & $\begin{array}{l}\text { Uso inapropiado } \\
\text { o innecesario }\end{array}$ \\
\hline $\begin{array}{l}\text { DNA-asa } \\
\text { recombinante } \\
\text { nebulizada }\end{array}$ & & & Uso inapropiado \\
\hline $\begin{array}{l}\text { Interferón } \\
\text { intramuscular }\end{array}$ & & & Uso inapropiado \\
\hline $\begin{array}{l}\text { Furosemida } \\
\text { nebulizada }\end{array}$ & & & Uso inapropiado \\
\hline \multicolumn{4}{|c|}{ Prevención } \\
\hline \multicolumn{4}{|c|}{ a) Prevención de sibilantes persistentes postbronquiolitis } \\
\hline Corticoides inhalados & & & Uso inapropiado \\
\hline Corticoides orales & & & Uso inapropiado \\
\hline Antileukotrienos & & & Uso inapropiado \\
\hline \multicolumn{4}{|c|}{ b) Prevención de BA por VRS } \\
\hline $\begin{array}{l}\text { Inmunoglobulina } \\
\text { intravenosa frente } \\
\text { VRS }\end{array}$ & & & No recomendado \\
\hline Palivizumab & & $\begin{array}{l}\text { Uso restringido } \\
\text { en prematuros y cardiópatas } \\
\text { de alto riesgo }\end{array}$ & $\begin{array}{l}\text { No recomendado } \\
\text { en otros escenarios }\end{array}$ \\
\hline
\end{tabular}

BA: bronquiolitis aguda; UCI: Unidad de Cuidados Intensivos; VRS: virus respiratorio sincitial: IV: vía intravenosa.

- Desde el punto de vista terapéutico únicamente se consideran dos intervenciones como de primera elección: el suplemento de oxígeno en los lactantes con BA con una $\mathrm{SatO}_{2}<92 \%$ y/o dificultad respiratoria grave, así como la aplicación de heliox en la Unidad de Cuidados Intensivos (UCl) en pacientes con compromiso respiratorio por BA grave.

Existe evidencia suficiente sobre la falta de eficacia de la mayoría de las intervenciones ensayadas en la BA. Al margen de la oxigenoterapia, la fluidoterapia, la aspiración de secreciones y la asistencia respiratoria, pocas opciones terapéuticas resultarán beneficiosas. Solo en los casos moderado-graves estaría justificado el ensayo con broncodilatadores inhalados (salbutamol o adrenalina) con suero salino hipertónico o sin él. En casos con fracaso respiratorio podrían emplearse heliox y técnicas de 
ventilación no invasiva, en pacientes con apneas se debe valorar el uso de metilxantinas, y en pacientes graves intubados el uso de surfactante.

El resto de intervenciones terapéuticas (salbutamol oral, adrenalina subcutánea, bromuro de ipatropio, corticoides inhalados, orales o sistémicos, antibióticos, inmunoglobulina intravenosa (IV) o aerosolizada, óxido nítrico, fisioterapia respiratoria, etc.) se consideran como uso inapropiado o innecesario. Sin embargo, los datos de campo de nuestro estudio indican que el tratamiento es inadecuado en el $43 \%$ de los pacientes con BA en fase aguda y en el $57 \%$ en fase de mantenimiento ${ }^{31}$.

- Desde el punto de vista preventivo, ningún tratamiento se ha mostrado eficaz para evitar la persistencia o recurrencia de síntomas postbronquiolitis. En cuanto a la prevención de bronquiolitis, solo palivizumab reduce discretamente el riesgo de ingreso por infecciones respiratorias bajas por virus respiratorio sincitial, aunque por su elevado coste solo se justifica su uso en un re- ducido grupo de pacientes de alto riesgo (grandes prematuros y cardiopatías hemodinámicamente significativas). Resulta significativo que, de las 61 preguntas al Panel de Expertos en la Conferencia de Consenso ${ }^{1}$, fue posible el consenso sobre el nivel de evidencia y grado de recomendación en todas salvo en las tres preguntas relacionadas con la eficiencia del palivizumab en la prevención de BA.

Consideramos importante la difusión de esta tabla sobre la clasificación de la idoneidad de procedimientos terapéuticos y preventivos en la BA, máxime en una enfermedad muy prevalente, con alto consumo de recursos y una variabilidad en la práctica clínica no justificada. El desarrollo de la misma se ha elaborado siguiendo las premisas de la medicina basada en la evidencia, cuyo objetivo es conseguir una práctica clínica más eficaz, efectiva, eficiente y segura.

Disponemos de las pruebas científicas (la "evidencia"), pero ahora conviene difundirlas $y$, sobre todo, implementarlas en la práctica clínica. La pregunta que sobrevuela es: ¿Será posible modificar la variabilidad injustificada en el manejo de la BA? 


\section{Bibliografía}

1. American Academy of Pediatrics (AAP). Subcommittee on Diagnosis and Management of Bronchiolitis. Diagnosis and management of bronchiolitis. Pediatrics. 2006;118:1774-93.

2. SIGN. Bronchiolitis in children. A national clinical guideline. Available at: htpp://www.sig nacuk. 2006.

3. Bronchiolitis Guideline Team, Cincinnati Children's Hospital Medical Center. Evidence based clinical practice guideline for medical management of bronchiolitis in infants year of age or less presenting with a first time episode. Cincinnati: Cincinnati Children's Hospital Medical Center; 2005. [En línea] [Consultado el 09/05/2009] Disponible en www.cincinnatichildrens.org/NR/ rdonlyres/0B7B99D7-DB3E-4186-B2FC71539E23 421E/0/bronchiolitisguideline.pdf.

4. National Guideline Clearinghouse (NGC). Guideline Synthesis. Prevention, diagnosis and treatment of pediatric bronchiolitis. [En línea] [Consultado el 09/05/2009] Disponible en: www.guidelinegov/Compare/comparisonaspx?f ile $=$ BRONCHIOLITIS1inc.

5. Hartling L, Wiebe N, Russell K, Patel $\mathrm{H}_{\text {, }}$ Klassen TP. Epinephrine for bronchiolitis. Cochrane Database Syst Rev. 2004(1):CD003123.

6. Spurling GK, Fonseka K, Doust J, Del Mar C. Antibiotics for bronchiolitis in children. Cochrane Database Syst Rev. 2007(1):CD005189.

7. Gadomski AM, Bhasale AL. Broncodilatadores para la bronquiolitis (Revisión Cochrane traducida). En: La Biblioteca Cochrane Plus, 2007 Número 4 Oxford: Update Software Ltd. Disponible en: http://www.update-softwarecom (Traducida de The Cochrane Library, 2007 Issue 4 Chichester, UK: John Wiley \& Sons, Ltd.).

8. Kellner JD, Ohlsson A, Gadomski AM, Wang EE. Bronchodilators for bronchiolitis.
Cochrane Database Syst Rev. 2000(2):CD00 1266.

9. Everard $M L$, Bara $A$, Kurian $M$, Elliott $T M$, Ducharme $F$, Mayowe V. Anticholinergic drugs for wheeze in children under the age of two years. Cochrane Database Syst Rev. 2005(3):CD 001279.

10. Everard $M L$, Bara $A$, Kurian $M$, Elliott $T M$, Ducharme F. Anticholinergic drugs for wheeze in children under the age of two years. Cochrane Database Syst Rev. 2002(1):CD001279.

11. Patel $H$, Platt $R$, Lozano J. WITHDRAWN: Glucocorticoids for acute viral bronchiolitis in infants and young children. Cochrane Database Syst Rev. 2008(1):CD004878.

12. Patel $H$, Platt $R$, Lozano JM, Wang EE. Glucocorticoids for acute viral bronchiolitis in infants and young children. Cochrane Database Syst Rev. 2004(3):CD004878.

13. McKean M, Ducharme F. Inhaled steroids for episodic viral wheeze of childhood. Cochrane Database Syst Rev. 2000(2):CD001107.

14. Blom D, Ermers M, Bont $L$, Van Aalderen WM, Van Woensel JB. Inhaled corticosteroids during acute bronchiolitis in the prevention of post-bronchiolitic wheezing. Cochrane Database Syst Rev. 2007(1):CD004881.

15. Perrotta C, Ortiz Z, Roque M. Chest physiotherapy for acute bronchiolitis in paediatric patients between 0 and 24 months old. Cochrane Database Syst Rev. 2007(1):CD004873.

16. Perrotta C, Ortiz Z, Roque M. Chest physiotherapy for acute bronchiolitis in paediatric patients between 0 and 24 months old. Cochrane Database Syst Rev. 2005(2):CD004 873.

17. Wang EE, Tang NK. WITHDRAWN: Immunoglobulin for preventing respiratory syncytial virus infection. Cochrane Database Syst Rev. 2006(3):CD001725. 
18. Wang EE, Tang NK. Immunoglobulin for preventing respiratory syncytial virus infection. Cochrane Database Syst Rev. 2000(2):CD00 1725.

19. Fuller $\mathrm{H}$, Del Mar C. Immunoglobulin treatment for respiratory syncytial virus infection. Cochrane Database Syst Rev. 2006(4):CD0048 83.

20. Ventre K, Randolph AG. Ribavirin for respiratory syncytial virus infection of the lower respiratory tract in infants and young children. Cochrane Database Syst Rev. 2007(1):CD000 181.

21. Ventre K, Randolph A. Ribavirin for respiratory syncytial virus infection of the lower respiratory tract in infants and young children. Cochrane Database Syst Rev. 2004(4):CD000181.

22. Zhang L, Mendoza-Sasi RA, Wainwright C, Klassen TP. Nebulized hypertonic saline solution for acute bronchiolitis in infants. Cochrane Database of Systematic Reviews Isue 4 Art No: CD006458 DOI: 101002/14651858CD0064 58pub2. 2008.

23. Ventre K, Haroon M, Davison C. Surfactant therapy for bronchiolitis in critically ill infants. Cochrane Database Syst Rev. 2006;3:CD 005150.

24. González de Dios J, Ochoa Sangrador C y Grupo de Revisión y Panel de Expertos de la Conferencia de Consenso del Proyecto aBREVIADo (BRonquiolitis-Estudio de Variabilidad Idoneidad y ADecuación). Conferencia de Consenso sobre bronquiolitis aguda (I): metodología y recomendaciones. An Pediatr (Barc). 2010;72: 221.e1-33.

25. Ochoa Sangrador C, González de Dios J y Grupo de Revisión del Proyecto aBREVIADo (BRonquiolitis-Estudio de Variabilidad Idoneidad y ADecuación). Conferencia de Consenso sobre bronquiolitis aguda (II): epidemiología de la bronquiolitis aguda. Revisión de la evidencia científica. An Pediatr (Barc). 2010;72:222.e126.

26. Ochoa Sangrador C, González de Dios J y Grupo de Revisión del Proyecto aBREVIADo (BRonquiolitis-Estudio de Variabilidad Idoneidad y ADecuación). Conferencia de Consenso sobre bronquiolitis aguda (III): diagnóstico en la bronquiolitis aguda. Revisión de la evidencia científica. An Pediatr (Barc). 2010;72:284.e1-23.

27. González de Dios J, Ochoa Sangrador C y Grupo de Revisión del Proyecto aBREVIADo (BRonquiolitis-Estudio de Variabilidad Idoneidad y ADecuación). Conferencia de Consenso sobre bronquiolitis aguda (IV): tratamiento de la bronquiolitis aguda. Revisión de la evidencia científica. An Pediatr (Barc). 2010;72:285.e1-42.

28. González de Dios J, Ochoa Sangrador C y Grupo de Revisión del Proyecto aBREVIADo (BRonquiolitis-Estudio de Variabilidad Idoneidad y ADecuación). Conferencia de Consenso sobre bronquiolitis aguda (V): prevención de la bronquiolitis aguda. Revisión de la evidencia científica. An Pediatr (Barc). 2010;72:353.e1-26.

29. Ochoa Sangrador C, González de Dios J y Grupo de Revisión del Proyecto aBREVIADo (BRonquiolitis-Estudio de Variabilidad Idoneidad, Adecuación ADecuación). Conferencia de Consenso sobre bronquiolitis aguda (VI): pronóstico en la bronquiolitis aguda. Revisión de la evidencia científica. An Pediatr (Barc). 2010;72: 354.e1-34.

30. González de Dios, Ochoa Sangrador C. Manejo diagnóstico y terapéutico de la bronquiolitis aguda. GuiaSalud. [En línea] [Consultado el 18/10/2010] Disponible en www.guiasa lud.es.

31. González de Dios J, Ochoa Sangrador C, y Grupo Investigador del Proyecto aBREVIADo (BRonquiolitis-Estudio de Variabilidad, Idoneidad y ADecuación). Estudio de variabilidad en el ma- 
nejo de la bronquiolitis aguda en España en relación con la edad de los pacientes. An Pediatr (Barc). 2010;72:4-18.

32. Figueras Aloy J, López Sastre J, Medrano López C, Bermúdez-Cañete Fernández R, Fernández Pineda L, Bonillo Perales A et al. Consenso multidisciplinar español sobre la profilaxis de la infección respiratoria pediátrica por virus respiratorio sincitial. An Pediatr (Barc). 2008;69:63-71.

33. Castro-Rodríguez JA, Rodrigo GJ. Efficacy of inhaled corticosteroids in infants and preschoolers with recurrent wheezing and asthma: a systematic review with meta-analysis. Pediatrics. 2009;123:e519-25.

34. Grupo de Hospitales Benazuza. Quimioprofilaxis con palivizumab en Andalucía. Resultados de la epidemia de VRS 2000-2001. An Esp Pediatr. 2002;56:293-7.

35. Groothuis J, Bauman J, Malinoski F, Eggleston M. Strategies for prevention of RSV nosocomial infection. J Perinatol. 2008;28:319-23.

36. Kimpen JL, Schaad UB. Treatment of respiratory syncytial virus bronchiolitis: 1995 poll of members of the European Society for Paediatric Infectious Diseases. Pediatr Infect Dis J. 1997; 16:479-81.
37. New grades for recommendations from the Canadian Task Force on Preventive Health Care. CMAJ. 2003;169:207-8.

38. Atkins $D$, Best $D$, Briss PA, Eccles $M$, FalckYtter $Y$, Flottorp $S$ et al. Grading quality of evidence and strength of recommendations. BMJ. 2004;328:1490.

39. Guyatt $G H$, Oxman AD, Vist GE, Kunz R, Falck-Ytter $Y$, Alonso-Coello $P$ et al. GRADE: an emerging consensus on rating quality of evidence and strength of recommendations. BMJ. 2008;336:924-6.

40. Guyatt $G H$, Oxman AD, Kunz R, Vist GE, Falck-Ytter $Y$, Schunemann HJ. What is "quality of evidence" and why is it important to clinicians? BMJ. 2008;336:995-8.

41. Schunemann HJ, Oxman AD, Brozek J, Glasziou P, Jaeschke R, Vist GE et al. Grading quality of evidence and strength of recommendations for diagnostic tests and strategies. BMJ. 2008;336:1106-10.

42. Landau LI. Current pharmacological treatments for bronchiolitis are useless. The case for the con's. Paediatr Respir Rev. 2006;7(Suppl. 1):S101-3. 